\title{
Percepção das mulheres no climatério em relação à sexualidade, à prevenção de doenças sexualmente transmissíveis e à qualidade da assistência pelos profissionais da saúde
}

\author{
Perception of climacteric women in relation to sexuality, prevention of Sexually \\ Transmitted diseases and quality of care by health professionals
}

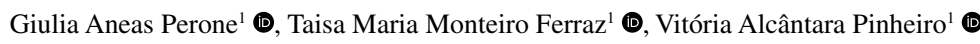

Ruth Bernarda Riveros Jeneral ${ }^{1}$

\begin{abstract}
RESUMO
Introdução: A fase climatérica, além de apresentar diversas alterações fisiológicas decorrentes do declínio das concentrações hormonais que interferem na qualidade de vida, sexualidade e autopercepção, ainda carrega os preconceitos e tabus criados pela sociedade. Objetivo: Identificar a percepção das mulheres na fase do climatério em relação aos sintomas apresentados, à sua sexualidade, ao uso de preservativo para prevenção de doenças sexualmente transmissíveis e à qualidade da assistência prestada pelos profissionais da saúde nessa fase. Metodologia: Trata-se de uma pesquisa descritiva com análise qualitativa, que incluiu 15 mulheres na faixa etária de 40 a 65 anos em condições de comunicação. Para a coleta de dados foi realizada uma entrevista gravada em áudio com a questão norteadora: "Como você se sente nessa fase da vida em relação à sua sexualidade, às doenças sexualmente transmissíveis e à assistência do profissional da saúde?’. Para a análise dos dados foi utilizada a técnica do discurso do sujeito coletivo, que visa dar luz ao conjunto de individualidades semânticas componentes do imaginário social. Resultados: Das 15 entrevistas, foi possível identificar 11 ideias centrais e 11 discursos. Mulheres climatéricas na faixa de 54 a 63 anos; 98\% usam preservativos; 94\% têm vida sexual ativa e companheiros há mais de 10 anos. Considerações finais: Percebeu-se que a parte do objetivo que contemplava a qualidade da assistência prestada pelos profissionais de saúde foi inconclusiva. Assim sendo, não obtivemos resultado final para esse tema, por ser entendido como um aspecto mais abrangente. Palavras-chave: sexualidade; climatério; doenças sexualmente transmissíveis.
\end{abstract}

\begin{abstract}
Introduction: The climacteric phase, in addition to presenting several physiological changes due to the decline of hormonal concentrations that interfere with quality of life, sexuality and self perception, still carry the prejudices and taboos created by society. Objective: To identify the perception of women in the climacteric phase regarding the symptoms presented, their sexuality, the use of condoms for the prevention of Sexually Transmitted Diseases and the quality of care provided by health professionals in this phase. Methodology: This is a descriptive research with qualitative analysis, which included 15 women aged from 40 to 65 years in conditions of communication. For data collection, a recorded audio interview was conducted with the guiding question: "How do you feel at this stage of life regarding your sexuality, Sexually Transmitted Diseases and the assistance of health professionals?". The Technique of Discourse of the Collective Subject was used to analyze the data, aiming to give light to the set of semantic individualities of the social imaginary. Results: From the 15 interviews, it was possible to identify 11 central ideas and 11 discourses. Climacteric women in the 54 to 63 -year age range; $98 \%$ use condoms; 94\% have been sexually active and with partners for more than 10 years. Final considerations: It was noticed that the part of the objective that contemplated the quality of care provided by health professionals was inconclusive. Therefore, we did not obtain an end result for this topic, because it is understood as a more comprehensive aspect.
\end{abstract}

Keywords: sexuality; climacteric; sexually transmitted diseases.

'Pontifícia Universidade Católica de São Paulo, Faculdade de Ciências Médicas e da Saúde - Sorocaba (SP), Brasil.

Autor correspondente: Ruth Bernarda Riveros Jeneral - Pontifícia Universidade Católica de São Paulo, Faculdade de Ciências

Médicas e da Saúde, Departamento de Enfermagem - Rua Joubert Wey, 290 - CEP: 18030-070 - Sorocaba (SP), Brasil -

E-mail: adoru@terra.com.br

Recebido em 05/12/2017 - Aceito para publicação em 04/10/2018. 


\section{INTRODUÇÃO}

O climatério é definido pela Organização Mundial da Saúde (OMS) como uma fase biológica da vida, e não um processo patológico, que compreende a transição da vida reprodutiva para a vida não reprodutiva da mulher e inicia entre os 35 e 40 anos, prolongando-se até os 65 anos de idade..$^{1,2}$ A fase não reprodutiva é caracterizada pela diminuição gradual da produção hormonal. O conceito de menopausa se entende como o último ciclo menstrual, após ter passado 12 meses da sua ocorrência. A carência hormonal, decorrente do climatério, é responsável pelas alterações fisiológicas no organismo da mulher, como por exemplo, as modificações significativas nos órgãos genitais internos e externos, que podem influenciar a resposta sexual. ${ }^{2}$ Geralmente, 50 a $70 \%$ das mulheres apresentam sinais, sintomas e alterações emocionais nessa fase de vida, com destaque para ondas de calor ou fogachos, sudorese, irritabilidade, ansiedade, depressão, insônia, fraqueza, cefaleia e palpitações, acontecendo mais durante a noite. ${ }^{3,4}$ Essas alterações não provocam necessariamente a diminuição do prazer, mas podem influenciar diretamente na resposta sexual, tornando-a mais lenta, podendo causar insatisfação sexual. Em contrapartida, uma das queixas mais recorrentes da fase, que afeta diretamente a qualidade da vida sexual, é a dor durante o ato sexual, denominada dispareunia, que pode decorrer do ressecamento vaginal. ${ }^{5}$

Entende-se que a assistência à mulher no climatério é uma das prioridades das políticas públicas de saúde direcionadas às mulheres, muito embora elas ainda estejam focadas demasiadamente no diagnóstico e tratamento das queixas clínicas apresentadas pelas mulheres, acarretando, com isso, a medicalização e a não valorização das queixas subjetivas, tais como: insatisfação sexual, medo em relação à falta de desejo, sensação de culpa diante das alterações que ocorrem com o ambiente familiar, com o seu corpo e no relacionamento com o seu parceiro. A sexualidade é um aspecto importante na qualidade de vida nesse período, pois as alterações hormonais influenciam a libido das mulheres. Além disso, o tema "sexualidade", historicamente e até os dias atuais, tem sido construído em torno dos mitos, tabus e crenças que cada sociedade vive e/ou viveu numa determinada época. ${ }^{6}$

Os ciclos vitais são as fases do processo da existência humana; e é necessário que o indivíduo se adapte e dê novo significado aos acontecimentos, dando, assim, uma nova configuração para cada fase de sua vida. ${ }^{7}$ Quando associado à sexualidade, torna-se um assunto ainda mais delicado, no qual podemos observar a herança baseada nos padrões julgados adequados, referentes a fatores sociais, políticos, religiosos e culturais. ${ }^{8}$

Sobre o processo de envelhecimento da mulher, encontra-se, por exemplo, a ideia errônea de que a mulher, ao envelhecer, se torna isenta de interesse sexual e que deve seguir o padrão de beleza e comportamento impostos pela mídia e sociedade. Assim, quando analisados os fatores holísticos de condições de vida, história reprodutiva, carga de trabalho, hábitos alimentares, dificuldade de acesso aos serviços de saúde, assim como outros conflitos socioeconômicos, culturais e espirituais associados aos citados aspectos do período climatérico, se observa tendência a desenvolver depressão, baixa autoestima, sensação de inutilidade, além das doenças crônicas e psicossomáticas, entre outras. ${ }^{1,7,8} \mathrm{~A}$ pouca abordagem do assunto em sua forma correta no ambiente assistencial, as hipóteses errôneas criadas pela sociedade e, consequentemente, pelos profissionais da saúde fazem com que haja uma barreira entre essas mulheres e o serviço de saúde, deixando-as sem amparo, apoio e espaço para suas questões pessoais e sexuais. Dessa forma, é evidente que a falta de informação sobre o processo de envelhecimento contribui para a consolidação de velhos tabus e estímulo à criação de novos preconceitos.

Todavia, inúmeras mulheres, apesar desse cenário, continuam com a vida sexual ativa, tornando-se mais suscetíveis a contraírem doenças sexualmente transmissíveis (DSTs). Atualmente, a população idosa é a que mais cresce, contribuindo para que o envelhecimento se torne um fenômeno mundial. Segundo a projeção do Instituto Brasileiro de Geografia e Estatística (IBGE), a população idosa em 2017 será de 8,46\%; e em 2030, de 13,44\%. Dessa forma, o número de idosos com vida sexual ativa aumenta e, consequentemente, também cresce o número de casos de DSTs. ${ }^{910}$

Esse cenário observado pelos alunos durante a graduação em enfermagem demonstra a carência de abordagem holística e sensível por parte dos profissionais da saúde, o que nos incentivou à realização do estudo, pois se torna necessária a identificação dos problemas vivenciados no período do climatério para que sejam amenizados.

\section{OBJETIVO}

Identificar a percepção das mulheres na fase do climatério em relação aos sintomas apresentados, à sua sexualidade, ao uso de preservativo para prevenção de DSTs e à qualidade da assistência prestada pelos profissionais da saúde nessa fase.

\section{METODOLOGIA}

Tratou-se de uma pesquisa descritiva com análise qualitativa. O estudo foi desenvolvido junto às pacientes idosas da Unidade de Saúde do bairro do Éden, na cidade de Sorocaba, São Paulo. As entrevistadas assinaram o Termo de Consentimento Livre e Esclarecido, de acordo com a Resolução $\mathrm{n}^{\circ}$ 466/2012. ${ }^{11}$ Participaram 15 mulheres na fase do climatério, que tinham companheiro ou não. Para a coleta de dados, foi realizada uma entrevista gravada em áudio com a questão norteadora: "Como você se sente nessa fase da vida em relação à sua sexualidade, às doenças sexualmente transmissíveis e à assistência do profissional da saúde?". As 15 entrevistas realizadas foram gravadas pelas alunas e posteriormente os depoimentos foram transcritos e organizados mediante a análise e metodologia do discurso do sujeito coletivo (DSC) de Lefévre et al. ${ }^{12,13}$ Esse método consiste em um conjunto de procedimentos de tabulação e organização de dados discursivos provenientes de depoimentos reais. 


\section{RESULTADOS}

\section{Caracterização das participantes}

Foram entrevistadas 15 mulheres na fase do climatério, sendo o perfil predominante a faixa etária entre 54 e 63 anos de idade, significando que todas as mulheres se encontravam em período de climatério. No que se refere ao uso de preservativo, $98 \%$ das mulheres não fazem e nunca fizeram o uso de preservativo para a prevenção de DSTs. A média da idade na qual as mulheres entrevistadas entraram na fase do climatério é de 8,8 anos. Em média, $94 \%$ têm companheiro há mais de 10 anos e permanecem com vida sexual ativa. O grau de escolaridade dessas mulheres é inferior ao fundamental II completo, sendo $1 \%$ analfabetas e nenhuma com curso de graduação.

A seguir, as ideias centrais (IC) e DSC para a questão norteadora: "Como você se sente nessa fase da vida em relação à sua sexualidade, às doenças sexualmente transmissíveis e à assistência do profissional da saúde?"

IC 1: Como as mudanças físicas do climatério interferem na vida (S1, S2, S3, S4, S7, S9, S11, S12, S14, S15)

DSC 1: "Meu marido falava: Você está velha, não quero mais você, é ruim ver o corpo ficando velho... E quando falava pra mim que eu estava um pouco gorda eu falava pra ele, "você também está... Tenho dores horríveis quando faço sexo, não tenho mais lubrificação vaginal".

IC 2: Pendências e dúvidas em consulta médica ( $\mathrm{S}$ 1, S2, S4, S5, S6, S7, S9, S11)

DSC 2: "Eles não tocam no assunto não, as vezes é só alguma coisa por cima. Não dão chance para você conversar. Daí você fica com receio de perguntar... Falta mais atenção, explicação... Eles deviam ser mais abertos para a gente poder se abrir... Se você tem que falar, vai ter que vencer a vergonha porque eles não perguntam".

IC 3: Desejo sexual no casamento ( $\mathrm{S} 1, \mathrm{~S} 2, \mathrm{~S} 6, \mathrm{~S} 7, \mathrm{~S} 9$, S11, S13, S14, S15)

DSC 3: "É o mais importante no casamento. Se não tem, não tem jeito, a mulher fica triste... O casamento sem o sexo, você já sabe, não dá em nada mesmo. É sim, é importante para um casamento feliz... O sexo é sim importante, é igual ela disse, é uma forma de amar a pessoa".

IC 4: Vida sexual ativa no casamento ( $\mathrm{S} 1, \mathrm{~S} 3, \mathrm{~S} 4, \mathrm{~S} 7$, S8, S10, S11, S13, S14)

DSC 4: "Não faço sexo todos os dias não, mas a cada três dias, a cada dois dias sim... O casamento sem o sexo, você já sabe, não dá em nada mesmo".

IC 5: Perda da libido (S2, S5, S8, S11, S13, S15)

DSC 5: "Não perdi o desejo pelo meu marido, não tenho por ele e nem ninguém... Eu satisfazia a vontade dele porque tem aquele ditado: Quem não marca presença, abre concorrência. Eu não falava "eu vou fazer só para satisfazer sua vontade"... Não sinto, mas não quer dizer que eu estou morta".
C 6: Preconceito (S1, S4, S6, S7)

DSC 6: "Meu filho fala que não quer que eu arrume namorado de jeito nenhum... Tem gente que fala besteira, que a gente não vive a vida normal só porque envelheceu... Com certeza alguns jovens têm preconceito".

IC7: Falta de prevenção contra as DSTs (S1, S2, S3, S4, S5, S6, S8, S9, S10, S12, S13, S14, S15)

DSC 7: "Muda totalmente a sensação... Eu nunca usei camisinha e também nunca tive outro parceiro... É que a gente acaba confiando mais no marido em relação a doenças $e$ tudo isso né... No meu tempo ninguém falava nada sobre esse negócio de AIDS".

IC 8: Busca assistência profissional para melhoria da saúde sexual do casal idoso (S1 e S12, S15)

DSC 8: "Meu marido com uns 40 e poucos anos já foi ficando impotente, a gente ia tentando e não dava. Eu que procurei tratamento para ele, ia na farmácia pedir pro farmacêutico... Novamente sempre buscando cuidar da saúde... Eu sempre incentivei ele... Eu cheguei a ir no medico, fiz reposição hormonal".

IC 9: Vivência na menopausa (S1, S2, S3, S4, S6, S7, S8, S9, S10, S11, S13, S14, S15)

DSC 9: "Eu sinto muito calor, muita quentura, sinto aquele calorzão e depois para e vem o frio e eu me incomodo muito com a minha barriga, ela começou a crescer depois da menopausa... Eu virei uma seca, engordei, meu cabelo não está como antes e parece que fiquei até mais mal humorada... Eu senti os calorão, mais tristeza depois da menopausa, parece que tudo piora... Eu gostaria de voltar a sentir vontade... Sem contar que a gente fica mais seca né... Pra ter relação era horrível, comecei a evitar quase sempre".

IC 10: Nunca foi orientado sobre sexualidade (S1, S9, $\mathrm{S} 13, \mathrm{~S} 14, \mathrm{~S} 15)$

DSC 10: "Quando eu era moça, minha mãe nunca falou nada disso para nós, nada... Tinha vergonha de explicar essas coisas para os filhos... Eu casei tapada... Porque os pais não falavam".

IC 11: Sexualidade ensinada aos filhos quebrando o tabu (S1, S3, S5, S6, S7, S9, S15)

DSC 11: "Hoje eu falo até com as minhas netas... Mas a gente não foi muito de falar dessas coisas em casa não. Acho que é por causa da criação mesmo... Eu tinha vergonha de falar disso... Eu falhei muito de não explicar as coisas".

\section{DISCUSSÃO}

Analisando os resultados, verificamos que é possível fazer uma correlação entre a fase fisiológica e o social singular de cada mulher em fase de climatério.

Estudos semelhantes identificaram que se considera o climatério como uma fase da vida biológica da mulher, que 
representa a transição entre o período reprodutivo e o não reprodutivo, e não um processo patológico, ${ }^{14}$ ressaltando que se trata de um período de transição com paradigmas sociais, como o da mulher fértil para infértil.

As mulheres entrevistadas, na maioria, alegam que na fase do climatério ocorrem mudanças, principalmente os sintomas que a fase do climatério apresenta. A variação na aparência física dessas mulheres, os sentimentos vivenciados de uma forma mais intensa nessa fase, a perda da libido, a diminuição do desejo sexual e até mesmo da prática do sexo com seus parceiros são um conjunto de situações que inevitavelmente interferem na qualidade de suas vidas. Dessa forma, estudos mostram que nos últimos anos têm surgido indagações os sintomas climatéricos e a tendência ao comprometimento intenso ou não da qualidade de vida no climatério, além do hipoestrogenismo, estarem associados também a fatores psicossociais e culturais relacionados ao processo de envelhecimento ou, então, pela sua interação. ${ }^{15}$

Atualmente, a hipótese é de que a qualidade de vida no climatério é influenciada tanto pela presença dos sintomas decorrentes do declínio estrogênico como por fatores psicossociais e culturais ligados ao próprio processo de envelhecimento. ${ }^{15,16}$

Levando em conta os resultados do perfil das mulheres em climatério entrevistadas, vimos que, em relação ao estudo, nenhuma das mulheres cursou nível superior e uma delas é analfabeta.

Estudos mostram que a percepção da menopausa e de sua medicalização, disseminada pelo discurso médico, pelos laboratórios farmacêuticos, pela mídia e até por ramos do discurso feminista, tem como público-alvo uma mulher privilegiada social e economicamente, com tempo e dinheiro disponíveis para cumprir numerosos rituais de saúde e beleza atribuídos a ela: exercícios físicos, cremes e vitaminas, alimentação balanceada, entre outros. Essa percepção pressupõe que a menopausa e o envelhecimento se apresentam da mesma forma a todas as mulheres, negando sua individualidade e contextos socioeconômicos e culturais. ${ }^{17}$

Profissionais de saúde menosprezam sua existência quando deixam de perguntar, na anamnese, sobre suas práticas sexuais, como se esse tópico não fosse importante ou como se não existisse em tal faixa etária. Diante da conjuntura atual, é notável a importância do profissional de saúde na assistência ao idoso, expondo informações esclarecedoras sobre sua sexualidade através do estabelecimento de vínculo. Assim, torna-se fundamental a necessidade de estabelecer uma relação de confiança para que o idoso relate suas dúvidas e seus medos. Dessa forma, destaca-se a importância de uma assistência de qualidade para que essas mulheres em fase de climatério sejam atendidas de uma maneira adequada e ciente das mudanças que estão ocorrendo nessa fase da vida. Porém, algumas mulheres entrevistadas se queixaram de não ter espaço nas conversas médicas para tirar suas dúvidas referentes às mudanças da fase do climatério.
Ante a comprovações, à complexidade da síndrome climatérica e dos seus possíveis reflexos na qualidade de vida feminina, tem sido proposta uma nova abordagem, destacando a importância de uma escuta qualificada paralela às intervenções clínicas necessárias, de forma a permitir maior compreensão do processo crítico existencial envolvido, onde aspectos psicológicos relacionados ao envelhecer se mesclam com aqueles resultantes do esgotamento hormonal. ${ }^{18}$

$\mathrm{Na}$ vida das mulheres existem marcos visíveis no corpo físico que sinalizam fases ou passagens, tais como a menarca, a ruptura do hímen e a última menstruação. Apesar de tais marcos serem rubricados em cada cultura, é possível identificar um traço aparentemente comum e presente em diferentes sociedades e épocas históricas: a valorização da mulher na fase reprodutiva e a sua desvalorização na fase não reprodutiva. Conforme a Federação Brasileira das Sociedades de Ginecologia e Obstetrícia, há mulheres que apresentam redução da libido na pós-menopausa, cuja explicação está na redução de testosterona, não de estrogênio. No entanto, a queda da produção de estrogênio torna lenta a lubrificação vaginal; e causa atrofia vaginal. ${ }^{19}$

No caso da sexualidade, avalia-se que há preconceito, visto que o tema é marcado por tabus e mitos. Mesmo numa sociedade moderna atual em que vivemos, quando se trata desse assunto na fase idosa é mais evidente o preconceito. O climatério caracteriza-se pelo caráter multidimensional, ou seja, não é influenciada somente por fatores anatômicos e fisiológicos, como também por fatores psicossociais e culturais, além de relacionamentos interpessoais e experiências de vida. Isso nos alerta que temos que tornar a educação mais facilitadora para a quebra desse tabu, visto que, ${ }^{20} \mathrm{em}$ 2050, um quinto da população mundial será de idosos. ${ }^{21}$ Dessa forma, é imprescindível que haja reflexões sobre a problemática, uma vez que as pessoas acima de 60 anos têm o direito de exercer sua sexualidade; e cabe a todos nós respeitá-las na integralidade.

Bonança $^{22}$ afirma que nascemos como sujeitos sexuados e desfrutamos de sexo/sexualidade de maneira diferente de acordo com a etapa de nossa vida, mas infelizmente a sociedade como um todo, e as pessoas de modo individual, tendem a pensar que o sexo/sexualidade pertence ao mundo dos jovens, relegando os indivíduos da terceira idade ao amor platônico ou à abstinência sexual. ${ }^{22}$

Sobre o uso de preservativo, ficou evidente que a porcentagem das mulheres que fazem uso é muito baixa, resultado alarmante, pois dados epidemiológicos em publicações recentes sobre DSTs evidenciam aumento global nos idosos em diversos países. ${ }^{23}$ No Brasil, por exemplo, estimativas da OMS apontam que há aproximadamente 937 mil novas infecções de sífilis; 1,5 milhão de casos de gonorreia; e quase 2 milhões de casos de clamídia por ano. Entretanto, dados mais precisos sobre o índice de transmissão de DSTs, especificamente na população acima de 50 anos, são escassos, por não serem doenças de notificação compulsória. ${ }^{24}$ 


\section{CONSIDERAÇÕES FINAIS}

As mudanças que foram vivenciadas por essas mulheres devem ser livres de preconceitos no que tange à sexualidade e também ao uso do preservativo nas relações sexuais, visto que a maioria tem vida sexual ativa, assim sendo, chegaremos mais perto de uma assistência digna, sendo uma assistência integral, holística e com atuação significativa para o combate das DSTs.

O maior interesse da equipe de profissionais por investigar a qualidade da vida sexual ativa dessas mulheres entrevistadas e o maior espaço nas consultas, para que essas mulheres esclareçam todas as dúvidas existentes, foram os destaques nos relatos obtidos; e entendemos que esse fato aponta como sendo uma peçachave na melhora da atenção à fase discutida. Compreendemos que o maior benefício seria os profissionais estarem capacitados e livres de preconceitos para que o atendimento seja adequado, assim, fazendo com que haja benefício no enfrentamento do processo da fase climatérica, e com isso estabilize a qualidade de vida, não havendo desconhecimento dos sintomas e tendo alternativas para solucionar alguns deles com ajuda dos profissionais adequados.

Esta pesquisa contribui para ampliar a visão sobre as dificuldades enfrentadas pelas mulheres em fase de climatério, que vão desde a realidade que essas mulheres vivenciam com os sintomas até o fator psicológico singular que cada uma apresenta, as condições sociais e familiares que possam lhes ofertar apoio nessa fase climatérica que enfrentam. Esse cenário fica ainda mais complicado quando se depara com a falta de capacitação das equipes multidisciplinares de saúde referente à não informação adequada e necessária na assistência a esse público climatérico.

Por outro lado, os resultados deste trabalho foram de muita valia por terem propiciado uma reflexão sobre o papel da equipe de saúde, em especial o enfermeiro dentro da equipe multidisciplinar, que deveria atender a essas mulheres em fase de climatério com acolhimento humanizado, contando com a quebra do paradigma do preconceito para uma melhora significativa na assistência nesse período da vida da mulher, proporcionando às pacientes mais espaço para as orientações e aplicação do autocuidado.

Queremos, com essa pesquisa, que os alvos sejam tanto os órgãos formadores de profissionais da saúde quanto os profissionais já formados e profissionais atuantes na área. Pois, dessa forma contamos que, posteriormente, a publicação desta pesquisa em periódicos nacionais facilite o desempenho dos profissionais do amanhã.

Realizaremos, contudo, a devolutiva dos resultados coletados desta pesquisa numa reunião com os profissionais da equipe de saúde da Unidade de Saúde do Éden, a fim de que possam aprimorar a assistência à saúde com as informações obtidas, ajudando na visão integral da mulher.

\section{REFERÊNCIAS}

1. Valença CN, Nascimento Filho JM, Germano RM. Mulher no climatério: reflexões sobre o desejo sexual, beleza e feminilidade. Saúde Soc. 2010;19(2):273-85. http://doi.org/10.1590/S0104-12902010000200005

2. Brasil. Ministério da Saúde. Secretaria de Atenção à Saúde. Departamento de Ações Programáticas Estratégicas. Manual de atenção à mulher no climatério/ menopausa. Brasília: Ministério da Saúde; 2008.

3. De Lorenzi DRS, Catan LB, Moreira K, Ártico G. Assistência à mulher climatérica: novos paradigmas. Rev Bras Enferm. 2009;62(2):287-93. http://doi. org/10.1590/S0034-71672009000200019

4. Adashi EY, Berek JS, Hilard PA. Tratado de Ginecologia. Rio de Janeiro: Guanabara Koogan; 1998.

5. Silva JPL, Marcolino EC, Fernandes NMS, Moura IM, Araújo MZ. Representações do climatério e suas repercussões na vida da mulher: uma revisão sistemática. Anais CIEH. 2015;2(1):1-12.

6. Alves ERP, Costa AM, Bezerra SMMS, Nakano AMS, Cavalcanti AMTS, Dias MD. Climatério: a intensidade dos sintomas e o desempenho sexual. Texto Contexto Enferm. 2015;24(1):64-71. http://doi. org/10.1590/0104-07072015000590014

7. Oliveira DM, Jesus MCP, Merighi MAB. Climatério e sexualidade: a compreensão dessa interface por mulheres assistidas em grupo. Texto Contexto Enferm. 2008;17(3):519-26. http://doi.org/10.1590/ S0104-07072008000300013

8. Bernardo R, Cortina I. Sexualidade na terceira idade. Rev Enferm UNISA. 2012;13(1):74-8.

9. Instituto Brasileiro de Geografia e Estatística. Projeção da população do Brasil e das Unidades da Federação [Internet]. Instituto Brasileiro de Geografia e Estatística [acesso em 20 mar. 2017]. Disponível em: https://www. ibge.gov.br/apps/populacao/projecao/

10. Santos AFM, Assis M. Vulnerabilidade das idosas ao HIV/AIDS: despertar das políticas públicas e profissionais de saúde no contexto da atenção integral: revisão de literatura. Rev Bras Geriatr Gerontol. 2011;14(1):147-57. http://doi.org/10.1590/ S1809-98232011000100015

11. Brasil. Conselho Nacional de Saúde. Resolução no 466, de 12 de dezembro de 2012. Brasília: CNS; 2012.

12. Lefévre F, Lefévre AMC, Teixeira, JJV. O Discurso do Sujeito Coletivo: uma nova abordagem metodológica em pesquisa qualitativa. Caxias do Sul: EDUCS; 2000.

13. Lefévre F, Lefévre F. O Discurso do Sujeito Coletivo: um novo enfoque em pesquisa qualitativa (desdobramentos). Caxias do Sul: EDUCS; 2005.

14. Almeida AB. Reavaliando o climatério: enfoque atual e multidisciplinar. São Paulo: Atheneu; 2003.

15. Lorenzi DRS, Baracat EC, Saciloto B, Padilha Júnior I. Fatores associados à qualidade de vida após menopausa. Rev Assoc Méd Bras. 2006;52(5):312-7. http://doi. org/10.1590/S0104-42302006000500017 
16. Sociedade Brasileira de Climatério. Consenso brasileiro multidisciplinar de assistência à mulher climatérica. São Paulo: SOBRAC; 2003.

17. Trench B. A saúde da mulher: reflexões sobre o envelhecer. In: Litvoc J, Brito FC, editores. Envelhecimento: prevenção e promoção da saúde. São Paulo: Atheneu; 2004. p. 220-6.

18. De Lorenzi DRS, Baracat EC. Climatério e qualidade de vida. Femina. 2005;33(12):899-903.

19. Federação Brasileira de Ginecologia e Obstetrícia. Climatério: manual de orientação. São Paulo: FEBRASGO; 2004.

20. Lorenzi DRS, Saciloto B. Frequência da atividade sexual em mulheres menopausadas. Rev Assoc Méd Bras. 2006;52(4):256-60. http://doi.org/10.1590/ S0104-42302006000400027
21. World Health Organization. Envelhecimento ativo: uma política de saúde. Brasília: Organização Pan-Americana da Saúde; 2005.

22. Bonança P. Sexualidade e tabu na terceira idade [Internet]. 2008 [acesso em 20 mar. 2017]. Disponível em: https://www.webartigos.com/artigos/sexualidadee-tabu-na-terceira-idade/12189

23. Dornelas Neto J, Nakamura AS, Cortez LER, Yamaguchi MU. Doenças sexualmente transmissíveis em idosos: uma revisão sistemática. Ciênc Saúde Coletiva. 2015;20(12):3853-64. http://doi.org/10.1590/1413812320152012.17602014

24. Brasil. Ministério da Saúde. Departamento de DST, AIDS e Hepatites Virais. DST no Brasil [Internet]. [acesso em 16 out. 2017]. Disponível em: http://www. aids.gov.br/pt-br/publico-geral/o-que-sao-ist

\section{Como citar este artigo:}

Perone GA, Ferraz TMM, Pinheiro VA, Jeneral RBR. Percepção das mulheres no climatério em relação a sexualidade, a prevenção de doenças sexualmente transmissíveis e a qualidade da assistência pelos profissionais da saúde. Rev Fac Ciênc Méd Sorocaba. 2019;21(2):77-82. http://doi.org/10.23925/1984-4840.2019v21i2a7 